\title{
Análisis de las características Corriente-Voltaje (I-V) en Barreras Schottky de Silicio (p)-Oro
}

\section{Analysis of the Current-Voltage (I-V) characteristics of Silicon (p)-Gold Schottky Barriers}

ORTIZ-VÁZQUEZ, Jonathan†๋*, ORTIZ-SIMÓN, José Luis, VÁZQUEZ-MÉNDEZ, Gerardo y ROJOVELAZQUEZ, Gustavo Emilio

\begin{abstract}
${ }^{1}$ Instituto Tecnológico de Nuevo Laredo, Reforma 2007 Sur. Colonia Fundadores. Nuevo Laredo Tamaulipas, México ${ }^{2}$ Departamento de Ingeniería Eléctrica, Sección de Electrónica del Estado Sólido, CINVESTAV, Av. Instituto Politécnico Nacional 2508, AP: 14-740, 07000 México, Ciudad de México.

${ }^{3}$ Programa de Doctorado en Nanociencias y Nanotecnología, CINVESTAV-IPN, Av. Instituto Politécnico Nacional 2508, Ciudad de México, C.P. 07360, México
\end{abstract}

ID $1^{\text {er }}$ Autor: Jonathan, Ortiz-Vázquez / ORC ID: 0000-0003-1087-9306, Researcher ID Thomson: S-7037-2018, CVU CONACYT ID: 999613

ID $1^{\text {er }}$ Coautor: José Luis, Ortiz-Simón / ORC ID: 0000-0001-6548-3849, CVU CONACYT ID: 209883

ID $2^{\text {do }}$ Coautor: Gerardo, Vázquez-Méndez / ORC ID: 0000-0001-8041-3072, CVU CONACYT ID: 1015828

ID $3^{\text {er }}$ Coautor: Gustavo Emilio, Rojo-Velazquez / ORC ID: 0000-0002-7792-1436, CVU CONACYT ID: 26367

DOI: $10.35429 / J T D .2019 .10 .3 .1 .5$

Recibido: 10 de Marzo, 2019; Aceptado 30 de Junio, 2019

\section{Resumen}

El ancho de banda, el rendimiento, la alta capacidad de almacenamiento su ancho de banda y el bajo costo de fabricación, son características de las memorias de acceso aleatorio (RAM). Dentro de la clasificación de las RAM se hallan las FRAM (memoria de acceso ferro-aleatorio) y las MRAM (memorias magnetorresistivas de acceso aleatorio). Esta última, ya que el almacenamiento se debe a una orientación magnética y no a una orientación eléctrica, ha causado mucho interés entre la comunidad científica. En este trabajo se analiza las características corriente-voltaje (I-V) en barreras Schottky de Silicio-Oro aplicando un campo magnético externo sobre esa estructura.

\section{MRAM, Barreras Schottky, Campo MRAM, Schottky Barriers, Magnetic Field}

\begin{abstract}
The bandwidth, low cost, great storage capacity and performance are characteristics of the random Access Memories (RAM).Some kinds of RAM include ferro-random access memory (FRAM) and magnetoresistive random access memory (MRAM), the latter being of great interest to the scientific community since storage is achieved via magnetic orientation rather than electric orientation. In this paper, we analyze the effect of the application of an external magnetic field on the current-voltage (I-V) characteristics of Schottky barriers made of silicon-gold, to show the effects of magnetoresistance in dispositive semiconductors suited for use as memory storage.
\end{abstract}

Citación: ORTIZ-VÁZQUEZ, Jonathan, ORTIZ-SIMÓN, José Luis, VÁZQUEZ-MÉNDEZ, Gerardo y ROJOVELAZQUEZ, Gustavo Emilio. Análisis de las características Corriente-Voltaje (I-V) en Barreras Schottky de Silicio (p)Oro. Revista del Desarrollo Tecnológico. 2019. 3-10: 1-5

\footnotetext{
* Correspondencia del Autor (Correo electrónico: j.o.vazquez1996@gmail.com)

$\uparrow$ Investigador contribuyendo como primer autor.
} 


\section{Introducción}

Los efectos de la magnetorresistencia se conocen desde 1850, cuando lord Kelvin mostro que la aplicación de un campo magnético a un objeto metálico incrementa la resistencia eléctrica del objeto en una dirección y la reduce en dirección de la normal. Fue gracias a los descubrimientos de la magnetorresistencia gigante (GMR), por parte de Alberto Ferta y Peter Grunberg, y con los recientes avances de la nanotecnología, que se desarrolló la MRAM [1]. Por otra parte, Zhang et al., reportan efectos de magnetorresistencia gigante en heterouniones Schottky Al/Ge/Al considerada como magnetorresistencia de rectificación; esta consiste en aplicar una corriente alterna a la heterounión Schottky no magnética lo cual puede generar una corriente significativa continua y un voltaje de rectificación, esté puede variar al aplicar un campo magnético externo.

Otros dispositivos que han generado gran interés en el estudio de propiedades magnéticas son los diodos resonantes túnel (RTD), por ejemplo, Likovich y colaboradores estudian películas a base de dos capas ferromagnéticas a base de GaMnAs donde una actúa como una barrera formando un pozo cuántico (QW), generando una barrera túnel, dando como resultado un dispositivo magnético con efecto túnel.

En el presente trabajo se analiza la estructura $\mathrm{ZnO} / \mathrm{Ti} / \mathrm{ZnO}$ colocando contactos de $\mathrm{Al} y \mathrm{Au}$ mediante el método de magnetorresistencia de rectificación. Se muestra que lo que se ha hecho es un estudio de la espectroscopia por fotoemisión. Este trabajo busca establecer si existe la posibilidad de usar esta estructura para el desarrollo de memorias magnéticas con efecto de diodo túnel.

\section{Metodología}

Para fabricar las barreras Schottky con silicio se utilizaron fracciones rectangulares de silicio tipo-p de orientación (100). Sobre los fragmentos rectangulares de silicio se realizó la barrera Schottky con un contacto de oro; para completar el circuito se realizó un contacto óhmico de aluminio en forma circular. Estos detalles se ilustran en la Figura 1. Los metales se depositaron por evaporación térmica.
Para fabricar las estructuras con el arreglo de $\mathrm{ZnO} / \mathrm{Ti} / \mathrm{ZnO}$ las películas de $\mathrm{ZnO}$ se crecieron por la técnica de magnetrón sputtering, las películas metálicas se depositaron por evaporación térmica y por evaporación por cañón de electrones Para conocer las características eléctricas de las películas de $\mathrm{ZnO}$ se depositaron sobre ellas contactos de In/Au para realizar mediciones por el método de efecto Hall. Los crecimientos fueron realizados de la siguiente manera: en primera instancia se realizó el lavado de los substratos de vidrio con xileno y acetona grado CMOS, posteriormente se retiró los residuos de acetona con agua desionizada mediante baño ultrasónico, una vez finalizado el proceso de limpieza se prosiguió con los crecimientos correspondientes de la estructura de $\mathrm{ZnO} / \mathrm{Ti} / \mathrm{ZnO}$.

La primera película es de $20 \mathrm{~nm}$ de $\mathrm{ZnO}$ la cual nos sirve para reducir los efectos generados por el substrato utilizado, está fue depositada por RF-sputtering utilizando una potencia de 125 watts con un flujo de Ar de $10 \%$. La segunda película de la estructura es de Ti con un espesor de $5 \mathrm{~nm}$ y se depositó mediante un cañón de electrones de vacío "Edwards E306 system". Finalmente se depositó una película de $\mathrm{ZnO}$ de $100 \mathrm{~nm}$ por Rf-Sputtering utilizando las mismas condiciones que la primera película.

Para comprobar que la superficie de la muestra fuera homogénea se realizó análisis morfológico mediante microscopia electrónica de barrido (SEM) Así mismo, se realizó análisis composicional por composición química (EDS), para ello las muestra fue preparada con una capa superficial de $\mathrm{Au}$ de $20 \mathrm{~nm}$ de espesor, las mediciones se realizaron en el microscopio de barrido FE HRSEM Auriga 3916 mediante InLens. Por otra parte, para la medición de las propiedades eléctricas se colocaron contactos de In y Au por separado, posteriormente se realizó la medición de efecto Hall por el método Hallvan der Pauw utilizando una corriente de $1 \mathrm{~mA}$ y un campo magnético de -0.5 Testa (T) a $0.5 \mathrm{~T}$.

Para analizar el efecto de rectificación en curvas I-V se colocaron contactos de In y $\mathrm{Au}$ como se muestran en la figura 1, sobre la estructura de $\mathrm{ZnO} / \mathrm{Ti} / \mathrm{ZnO}$. Las mediciones de las curvas I-V se realizaron tanto en polarización inversa como directa con voltajes en el rango de $-0.5 \mathrm{~V}$ a $0.5 \mathrm{~V}$. 


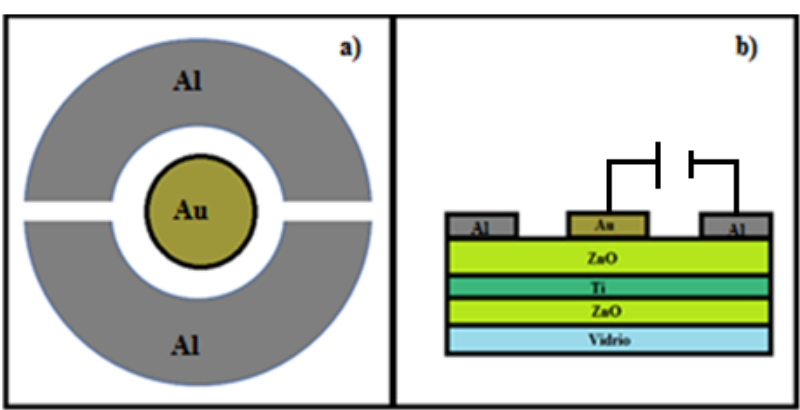

Figura 1 Esquema de contactos para mediciones de magnetorresistencia de rectificación. a) vista en plano, b) vista en sección transversal de la estructura $\mathrm{ZnO} / \mathrm{Ti} / \mathrm{ZnO}$

\section{Resultados}

a)

\section{Caracteristicas de las superficies de las muestras}

Las superficies de las muestras de silicio son extraordinariamente lisas de tal manera que al analizarlas al microscopio no se observa rugosidad alguna, pueden describirse como superficies con terminación a espejo. Sobre ellas se forman las metalizaciones con la geometria mostrada por en Figura 2.

En la figura 2, se muestra la morfológia de las estructura con $\mathrm{ZnO}$. Se indica tambien la composición quimica de la superficie de la estructura de $\mathrm{ZnO} / \mathrm{Ti} / \mathrm{ZnO}$. Si nota la presencia de los elementos principales, Ti, Zn y Oxígeno. En la figura 2 a) se puede observar que la muestra tiene una superficie con distribución homogenea con particulas menores a $100 \mathrm{~nm}$. Por otra parte en la figura $2 b$, se muestra el analisis composicional donde se identifico la presencia de $\mathrm{Zn}$, Ti y $\mathrm{O}$ con una concentración $\sim 32, \sim 2$ y $\sim 66$ en porcentaje atomico respectivamente.

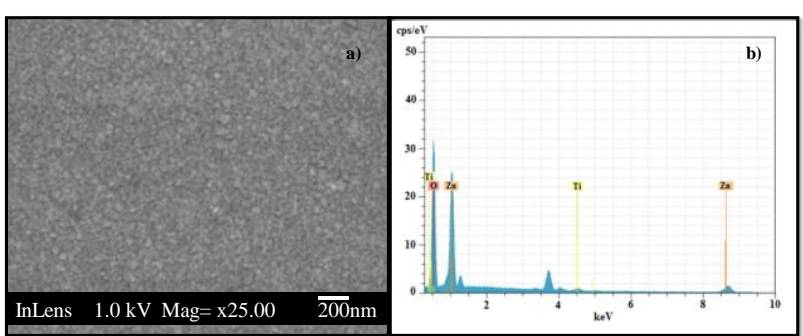

Figura 2 Micrografias de la superficie de la estructura de $\mathrm{ZnO} / \mathrm{Ti} / \mathrm{ZnO}$ obtenidas por SEM a), analisis de composición química obtenido mediante EDS b)

\section{b) Propiedades electricas de las peliculas semiconductoras}

Las propiedades electricas de la estructura de $\mathrm{ZnO} / \mathrm{Ti} / \mathrm{ZnO}$ (J-1) contenidos en la Tabla 1, se obtuvieron por el método de Hall-van der Pauw a temperatura ambiente (J1-300 K) y a $77 \mathrm{~K}$ (J1$77 \mathrm{~K})$. En la Tabla 1 se muestran los resultados de las propiedades electricas de la estructura realizada fueron comparados con los de una muestra cristalina de $\mathrm{ZnO}$ tipo-n. En este sentido se puede observar que la estructura es tipo-n, presentando una concentración de portadores de $\approx 10^{21} \mathrm{~cm}^{-3}$ y baja resistividad en comparación con el cristal de $\mathrm{ZnO}$ sin impurificar, estos efectos pueden ser atribuidos a la presencia de la capa intermedia de Ti.

\begin{tabular}{|c|c|c|c|}
\hline Muestra & $\begin{array}{l}\text { Resistividad } \\
(\Omega \mathrm{cm})\end{array}$ & $\begin{array}{l}\text { Movilidad } \\
\left(\mathrm{cm}^{2} / \mathrm{Vs}\right)\end{array}$ & $\begin{array}{c}\text { Concentración } \\
\left(\mathrm{cm}^{-3}\right)\end{array}$ \\
\hline $\begin{array}{l}\text { Cristal } \\
\mathrm{ZnO}\end{array}$ & $3.13 \times 10^{-2}$ & 97.960 & $-2 \times 10^{18}$ \\
\hline $\begin{array}{l}\mathrm{J}-1, \\
300 \mathrm{~K}\end{array}$ & $4.91 \times 10^{-3}$ & 1.2656 & $-1 \times 10^{21}$ \\
\hline $\mathrm{J}-1,77 \mathrm{~K}$ & $5.99 \times 10^{-3}$ & $9.09 \times 10^{-2}$ & $-7 \times 10^{21}$ \\
\hline
\end{tabular}

Tabla 1 Propiedades eléctricas de la estructura de $\mathrm{ZnO} / \mathrm{Ti} / \mathrm{ZnO}$ por el método Hall-van der Pauw utilizando un campo magnético $0.55 \mathrm{~T}$

\section{c) Características I-V de las Barreras Metal-Semiconductor}

Para facilitar la descripción del comportamiento de las Barreras Metal-Semiconductor y de los resultados experimentales obtenidos es necesario mostrar su constitución física y la representación de la energía que poseen los electrones que se desplazan en ella cuando se aplica una diferencia de potencial externa. El esquema correspondiente se ve en la Figura 3. El detalle básico es la barrera de potencial que se establece en el semiconductor señalada como Barrera Schottky; esta barrera se puede modular al aplicar un potencial externo, si el potencial es positivo la barrera aumenta y en caso contrario la barrera disminuye.

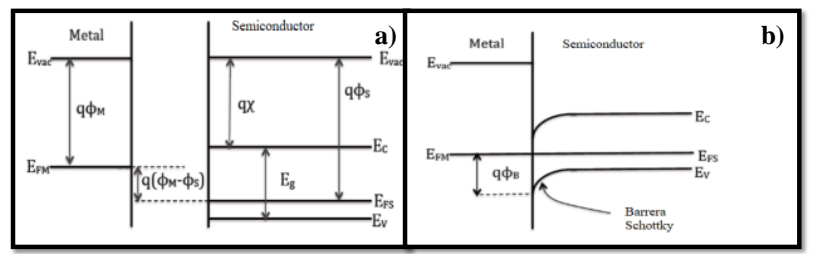

Figura 3 Diagrama de bandas de energía de la Barrera Schottky. a). - Metal y semiconductor separados, b) Metal y semiconductor en contacto mostrando la barrera de potencial efectiva 
Como resultado de la polarización se establece una corriente que depende de las características físicas de la estructura. En la Figura 3 se escribe la Ley de la Barrera Schottky que contiene dos términos, el prefactor $\mathrm{I}_{\mathrm{sat}} \mathrm{O}$ corriente de saturación, y el término entre paréntesis, que describe el comportamiento de la barrera en polarización directa y en polarización inversa. En el esquema de la Figura 4-a) se ilustra el significado de la corriente de saturación y la variación esperada en la corriente con voltajes de polarización cercanos a cero volts.

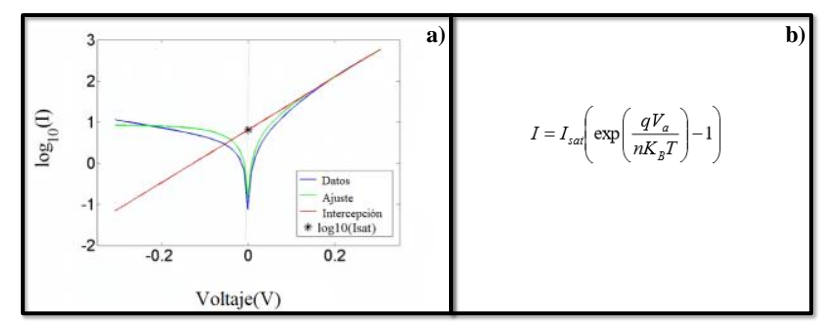

Figura 4 Característica Corriente-Voltaje de una Barrera Schottky. a)- Variación de la corriente en la Barrera para voltajes de polarización próximos a cero volts. b). - Ley de la Barrera Schottky

\section{d) Resultados Experimentales}

La característica I-V en la Barrera Schottky de Au-Silicio en ausencia de campo magnético y con un campo magnético aplicado de 0.5 Tesla se incluyen en la Figura 5.

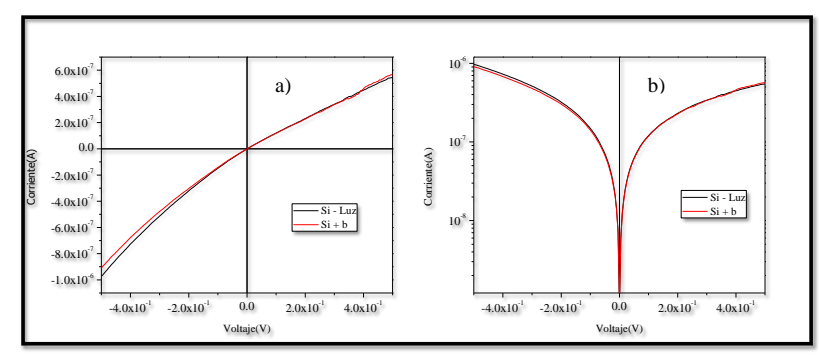

Figura 5 Curva I-V para una barrera Schottky sobre Si tipo-p a temperatura ambiente, sin campo magnético línea negra y con presencia de campo magnético de $0.5 \mathrm{~T}$ línea roja

La Fig. 5 a) corresponde a la característica I-V en escala lineal y la Fig. b) presenta los mismos resultados, pero en escala semi-logarítmica para hacer más claros los cambios que se producen. Un resultado relevante asociado al propósito de este trabajo es el efecto que se produce sobre las características I-V por la aplicación de un campo magnético.
La línea verde corresponde a la corriente que circula por la barrera sin campo magnético aplicado y la línea roja es la corriente eléctrica que fluye por la barrera cuando la estructura está polarizada y se aplica un magnético aplicado en dirección perpendicular a la superficie de la estructura. El efecto del campo magnético es que la corriente en la barrera Schottky disminuye; este resultado se puede explicar porque se produce un aumento en la resistencia por efecto del campo magnético aplicado correspondiente a la magnetorresistencia con el dispositivo en operación.

La característica I-V en la Barreras producida sobre las estructuras de varias capas se incluye en la Figura 6.
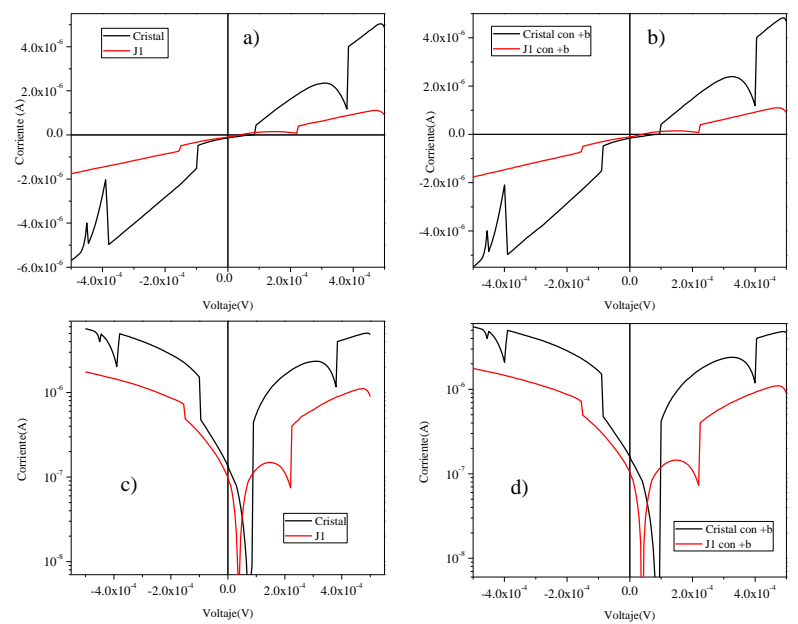

Figura 6 Curva I-V para un cristal de $\mathrm{ZnO}$ y la estructura de $\mathrm{ZnO} / \mathrm{Ti} / \mathrm{ZnO}(\mathrm{J} 1)$ a temperatura ambiente (a). Rectificación de magnetorresistencia en curvas IV, (b) en presencia de un campo magnético de $0.5 \mathrm{~T}$ (c y d) y sus representaciones logarítmicas

\section{Discusión de resultados}

De acuerdo al propósito del trabajo se busca dar una justificación a los cambios que se producen en la corriente de las estructuras cuando estas se exponen a un campo magnético. En la Figura 4 a) se presenta el comportamiento característico de las barreras Schottky de silicio en polarización directa e inversa con voltajes menores a $300 \mathrm{mV}$ sin campo magnético aplicado. Se observa el efecto de la barrera y se indica la corriente de saturación característica en los dispositivos. En las figuras 5 se presentan los datos obtenidos en la barrera Schottky que se fabricó, la corriente de saturación de la barrera fue de $2.0 \times 10^{-7} \mathrm{~A}$, de acuerdo a la literatura este valor es usual en los dispositivos de silicio. 
En esta figura se ve el efecto que produce la aplicación del campo magnético, la corriente en la barrera disminuye lo que indica que se manifiesta el efecto magneto resistivo, la corriente disminuye hasta $6.36 \times 10^{-8} \mathrm{~A}$, un factor de 3 .

\section{Conclusiones}

Mediante microscopia SEM se pudo observar la superficie de la estructura $\mathrm{J} 1 \quad(\mathrm{ZnO} / \mathrm{Ti} / \mathrm{ZnO})$ obtenida mediante magnetrón sputtering es homogénea, presentando partículas menores a $100 \mathrm{~nm}$. Asimismo, para corroborar la presencia de la capa intermedia de Ti se realizó análisis EDS obteniendo $\sim 2 \%$ en porcentaje atomico de Ti.

Los estudios de propiedades eléctricas muestran que la estructura J1es tipo-n y presentan una resistividad de $4.9 \times 10^{-3} \Omega \mathrm{cm}$, con concentración de portadores de $\approx 10^{21}$, con una movilidad del electrón de 1.26.

Se encontró que la aplicación del campo magnético en la barrera Schottky de silicio produce un cambio en la magneto resistencia que reduce la corriente en la estructura en un factor de 3 .

\section{Referencias}

Hanbicki A. T., Magno R., Cheng S. F.,. Park Y. D, Bracker A. S., and Jonker B. T. (2001) Nonvolatile reprogrammable logic elements using hybrid resonant tunneling diode-giant magnetoresistance circuits, Appl. Phys. Lett., vol. 79, no. 8, pp. 1190-1192,

Likovich E.et al., (2009) Magnetoresistance in an asymmetric Ga1-xMnxAs resonant tunneling diode, Phys. Rev. B - Condens. Matter Mater. Phys., vol. 80, no. 20, pp. 15-18,.

Slobodan Obradović1 N. D., Borivoje Milošević2 (2011) Magnetoresistive Random Access Memory, Handb. Spin Transp. Magn., vol. VII-2, pp. 196-206.

Zhang K.et al., (2015). Large rectification magnetoresistance in nonmagnetic $\mathrm{Al} / \mathrm{Ge} / \mathrm{Al}$ heterojunctions," Sci. Rep., vol. 5, pp. 1-6, 2015. 